\title{
A Method of Calculating Measuring Interval for Large-Size Straightness
}

\author{
Shengzhou Lu \\ line 1:Mechanical and Electrical Engineering \\ line 2: Harbin Institute of Technology \\ line 3: Harbin, China \\ line 4: e-mail: lupassion@163.com
}

\author{
Weishun Wang \\ line 1: Research and Development Center \\ line 2: Qiqihar Heavy CNC Equipment CORP. LTD \\ line 3: Qiqihar, China \\ line 4: e-mail: 13836260538@163.com
}

\author{
Zhongxi Shao \\ line 1:Mechanical and Electrical Engineering \\ line 2: Harbin Institute of Technology \\ line 3: Harbin, China \\ line 4: e-mail: shaozhongxi78@163.com
}

\author{
Hongya $\mathrm{Fu}$ \\ line 1:Mechanical and Electrical Engineering \\ line 2: Harbin Institute of Technology \\ line 3: Harbin, China \\ line 4: e-mail: hongyafu@hit.edu.cn
}

\begin{abstract}
Accurately straightness measuring has a direct relation to the promotion of the machine precision. In the process of straightness measurement, since the measuring interval has a great impact on the accuracy and efficiency of large-size straightness measurement, and there is no detailed measurement interval calculation method at present, it is necessary to make a deeply research to the measuring interval. This paper gives theoretical derivation to the measuring interval based on the Nyquist sampling theorem. Maximum frequency $M$ and the number of measuring points in one period $\mathrm{N}$ are determined based on measurement tolerance, and then calculate the optimal theoretical measuring interval. Finally, measure the straightess of the guide rail under diferent measuring interval including the optimal one. Validate the calculation method through the straightess measuring experiment. The experimental results show that the optimal theoretical measuring interval derived in this paper is suitable, which proves the correctness of the calculating method.
\end{abstract}

Keywords-Straightness; Measuring interval; Large-size; Accuracy; Optimal theoretical measuring interval.

\section{INTRODUCTION}

Straightness tolerance is one of the geometric tolerances which are specified in the national standard and the international organization for standardization (ISO), and it is mainly used to control the shape error of straight line in plane or space[1]. The accurate measurement and evaluation of the straightness error is very important to eligibility judgment and guarantee of geometry parts with the straightness tolerance requirements[2]. Straightness measurement usually adopts the method of equal intervals measurement to measure the measured object through the measuring interval. There isn't convincingly theoretical basis about the definition of the measuring interval in the related standard of country and enterprise[3], while the definition mostly depends on the experience of the technician[4][5]. The large measuring interval brings few measuring points, which will result in part of information losing that can cause the shape of the measured object cannot be accurately measured. Conversely, if the measuring interval is too small and the measuring points are too many, it will bring some interference information and useless data, especially for large-size straightness measurement, therefore this will cause low measuring efficiency and untrusted measuring results. So it is necessary to make a deeply theoretical analysis about how to choose the straightness measuring interval of largesize, and to choose suitable measuring interval in measurement of large-size under the premise of measurement requirement,aiming to improve the efficiency of straightness measurement and evaluation and to ensure the credibility of the measurement results.

\section{THE DETERMINATION OF MEASURING INTERVAL IN LARGE-SIZE STRAIGHTNESS MEASUREMENT}

\section{A. Nyquist sampling theorem}

At present, the theoretical basis of determining the measuring interval is the Nyquist sampling theorem at home and abroad [6], the content of the theorem is as follows: If the maximum frequency of a continuous single value analog is $\mathrm{M}$ and the sampling frequency is $2 \mathrm{M}$, it will completely determine the analog waveform. It means that there must be sampled at least twice times in one changing cycle of the maximum frequency. The sampling period now is presented by Eq.(1)

$$
\Delta<\frac{1}{2 M}
$$

where $\Delta$ and $M$ respectively stand for sampling period and the maximum frequency of single value analog. 
The measuring interval corresponds to the sampling period of the signal in the time domain[7]. By the Nyquist sampling theorem, the number of measuring points in total length $\mathrm{K}$ can be determined by the maximum frequency $\mathrm{M}$ and the number of sampling in one changing cycle $\mathrm{N}$ of the maximum frequency. With the total measurement length $\mathrm{L}$, the measuring interval can be determined as Eq.(2)

$$
\Delta=\frac{L}{K}=\frac{L}{M N}
$$

where $\mathrm{L}, \mathrm{K}, \mathrm{M}$ and $\mathrm{N}$ respectively represent total measuring length, theoretical optimum measurement points in total measurement length, the maximum frequency and the number of sampling $\mathrm{N}$ in one changing cycle of the maximum frequency $(N>2)$.

It is observed that the measuring interval $\Delta$ can be determined by total measurement length $\mathrm{L}$, the maximum frequency $\mathrm{M}$ of the signal of the surface shape of the measured guide rail and the number of measuring points $\mathrm{N}$ in one changing cycle of the maximum frequency. Figure.1 describes the calculation process of theoretical measuring interval of large-size straightness.

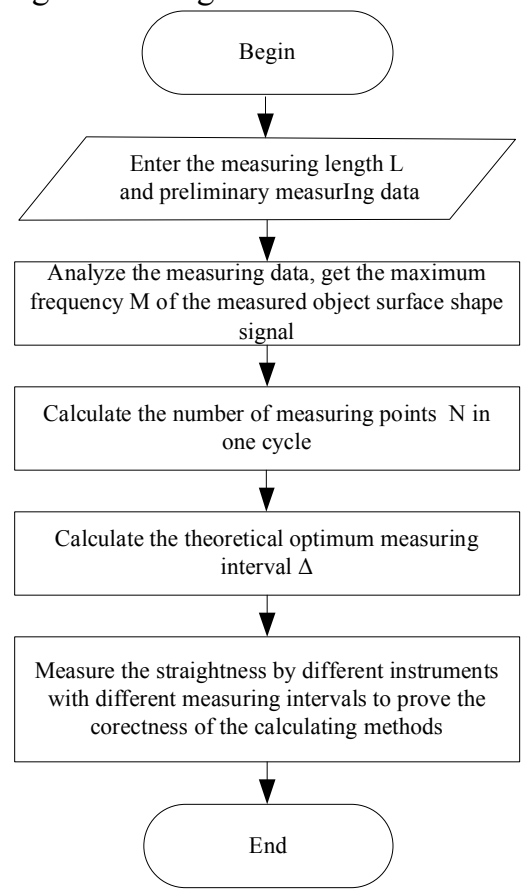

Figure.1 The calculation Process of Theoretical Measuring Interval of Large-size Straightness

\section{B. The determination of the maximum frequency $M$}

Since the determination of the maximum frequency of surface profile signal $\mathrm{M}$ is related with the measurement tolerance $\delta$, there needs to introduce the measurement tolerance $\delta$. The purpose of straightness measurement is to truly express the measured surface shape. The measurement errors from the measuring method and instrument will cause that the machining errors of the measured object cannot be accurately measured, so we should try to reduce measuring errors which mainly refers to errors from the method. In practice, the measurement method of equal interval is often used in straightness measurement, but the uncertainty of measuring interval brings the uncertainty of the position of measuring. The measuring errors from the method are mainly caused by mismatching between the measurement points and the extreme values points of the measured surface profile, and the measurement tolerance $\delta$ is used to restrict the measuring errors especially from measuring methods. Literature [8] points out that measurement tolerance $\delta$ relate with tolerance grade level and size of the measured object. By selecting the appropriate measurement tolerance $\delta$ based on the characteristics of the measured object, we can determine the maximum frequency $\mathrm{M}$ of the measured object surface shape of the signal.

The error signal of the surface profile in the measured guide rail contains geometrical form error v surface waviness and surface roughness. In terms of frequency and amplitude, the low frequency and large amplitude makes the geometrical form error be the major factor affecting the surface appearance. The surface roughness frequency is high and amplitude is small. The frequency and amplitude of the surface wave are between both the above[9]. In the actual measurement, because of the difference of measured surface, the maximum frequency $\mathrm{M}$ of surface profile signal is not the same. The specific way to determine the $\mathrm{M}$ is to find out the former $\mathrm{M}$ order harmonic components of the measured surface profile signal, then compare each order harmonic amplitude with the measurement tolerance $\delta$, find the maximum frequency $\mathrm{M}$ whose amplitude is above the measurement tolerance $\delta$.

In summary, according to the selected measuring interval determined by the total length $\mathrm{L}$ of the measured object and related national standards, measure the object and get the measurement data, and then modify the data through least square method to eliminate the influence of the installation inclination of the measured surface. Process the revised data by Fourier transform and data $\mathrm{X}(\mathrm{k})$ after Fourier transform which is described as Eq.(3)

$$
X(k)=D F T[x(i)]=\sum_{i=0}^{n-1} x(i) e^{\frac{-j 2 \pi i k}{n}}
$$

where $\mathrm{x}(\mathrm{i})$ and $\mathrm{n}$ respectively represent the original measurement data and the total number of measuring points.

Compare each order harmonic amplitude $\mathrm{C}_{\mathrm{i}}$ with the measurement tolerance $\delta$, find the harmonic $\mathrm{C}_{\mathrm{m}}$ whose amplitude is above the measurement tolerance $\delta$ and order is the highest, then the maximum frequency is $\mathrm{M}$.

\section{The determination of the number of meauring points in one cycle $N$}

In the process of straightness measurement,the extreme values ultimately determine the straightness error value. Any one periodic signal can be formed by a number of sinusoidal signals, so we can assume that the measured surface profile is a sine wave, its period is T and its amplitude is $a$ as Figure.2 describes, then we can see $\mathrm{B}$ and $\mathrm{D}$ is the determinant to straightness error value. As can be seen from Figure.2, the 
decrease of the measuring interval will make the measurement points much closer to the extreme values points and then finally decrease the deviation caused by the mismatching between the measurement points and the extreme values points. But the decrease of the measuring interval and the increasing of the measurement points reduce the measurement efficiency and increase the amount of calculation, there is need to discuss the optimum number of measuring points within one cycle N[10].

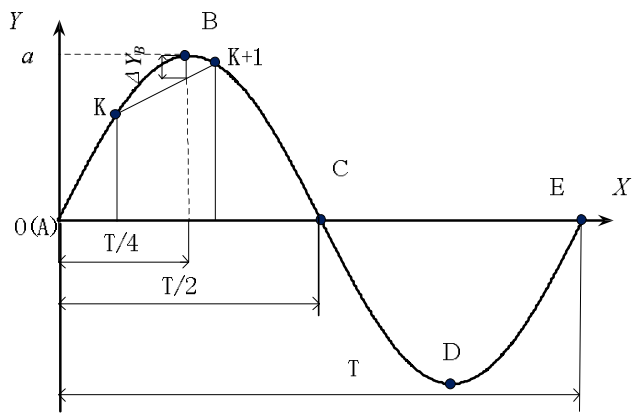

Figure.2 Relationship between Measurement Error and Measuring Position

Assume that the number of measuring points in one changing cycle of the maximum frequency on the measured surface is $\mathrm{N}$, the amplitude of the frequency component is $a$ and its period $T=2 \pi$, so the measuring interval is $2 \pi / N$. The measuring starting point is zero $\mathrm{A}$, and $\mathrm{B}$ fall between $\mathrm{K}$ and $\mathrm{K}+1(K=0,1,2 \ldots \ldots N)$, then the difference between $\mathrm{K}$ and $\mathrm{B} \Delta Y_{K}$ and $\mathrm{K}$ and $\mathrm{B} \Delta Y_{K+1}$ are determined as Eq.(4) and Eq.(5)

$$
\begin{aligned}
& \Delta Y_{K}=a-a \sin (2 \pi K / N) \\
& \Delta Y_{K+1}=a-a \sin (2 \pi(K+1) / N)
\end{aligned}
$$

In actual measurement, the position of the measured point is unknown. Assume that the actual positions of the measured points and the measuring point position deviation is $\Delta X$, then the difference of $B$ value and $K$ and $K+1$ point measurements are respectively determined as Eq.(6) and Eq.(7)

$$
\begin{aligned}
& \Delta Y_{K}=a-a \sin ((2 \pi K / \mathrm{N})+\Delta \mathrm{X}) \\
& \Delta Y_{K+1}=a-a \sin ((2 \pi(K+1) / \mathrm{N})+\Delta \mathrm{X})
\end{aligned}
$$

Point $\mathrm{B}$ is between point $\mathrm{K}$ and point $\mathrm{K}+1$, so when the actual position of measuring point changes, the variation trend of $\Delta \mathrm{Y}_{\mathrm{K}}$ and $\Delta \mathrm{Y}_{\mathrm{K}+1}$ is just the opposite, one increases, another reduces. It can be proved that the limitation of measurement error caused by the mismatching between the measurement points and the extreme values points. $\Delta \mathrm{Y}_{\mathrm{K}}=\Delta \mathrm{Y}_{\mathrm{K}+1}$, the change of the actual measurement point $\Delta \mathrm{X}$ is as Eq.(8)

$$
\Delta X=(N-4 K-2) \pi /(2 N)
$$

Take Eq.(8) into Eq.(6) and Eq.(7), the limit of measurement error near B is as Eq.(9)

$$
\Delta Y_{B \max }=a(1-\cos (\pi / \mathrm{N}))
$$

Similarly, the limit of measurement error near the extreme value point $\mathrm{D}$ is as Eq.(10)

$$
\Delta Y_{D \max }=a(1-\cos (\pi / \mathrm{N}))
$$

Therefore, the limit of straightness measurement error $\Delta Y_{\max }$ caused by mismatching between the measurement points and the extreme values points are determined as Eq.(11)

$$
\begin{aligned}
\Delta Y_{\max } & =\Delta Y_{B \max }+\Delta Y_{D \max } \\
& =2 a(1-\cos (\pi / \mathrm{N}))
\end{aligned}
$$

As can be seen from $\operatorname{Eq}(11)$, with the number of measuring points $\mathrm{N}$ increasing,limit error of straightness measurement gets smaller. Therefore, as long as we know the amplitude of the maximum frequency of the measured surface signal a, we can get the number of measuring in one cycle of the maximum frequency $\mathrm{N}$ with measurement tolerance $\delta$.

\section{Determination of straightness measuring interval $\Delta$}

Combine the maximum frequency $\mathrm{M}$ and the number of sampling in one changing cycle of the maximum frequency $\mathrm{N}$, we can get the number of measuring points $\mathrm{K}$ in the total measuring length which descibes the determination of theoretically optimal measuring points as Eq.(12)

$$
K=M N
$$

And theoretically optimal measuring interval in total measurement length is determined as Eq.(13)

$$
\Delta=\frac{L}{K}=\frac{L}{M N}
$$

\section{EXPERIMENTAL VERIFICATION TO THE CALCULATION METHOD FOR MEASURING INTERVAL OF LARGE STRAIGHTNESS}

Theoretically optimal measuring interval can be deduced according to the large-size straightness measuring interval calculation method with the original experimental data obtained. Validate the theoretically optimal measuring interval derived in this paper is reasonable, through measuring the straightness by different measuring interval including the optimal one.

The measuring object in experiment is straightness within the vertical plane of the $5 \mathrm{~m}$ guide rail whose accuracy index $\mathrm{T}$ is $0.02 \mathrm{~mm}$ and the measuring instruments is Renishaw XL-80 laser interferometer. In order to eliminate the effect of the tilt of the rail to straightness measurements, use the least squares method to assess the straightness. It is recommended that the measurement tolerance $\delta$ in the calculation can be $\mathrm{T} / 5 \sim \mathrm{T} / 10$. 


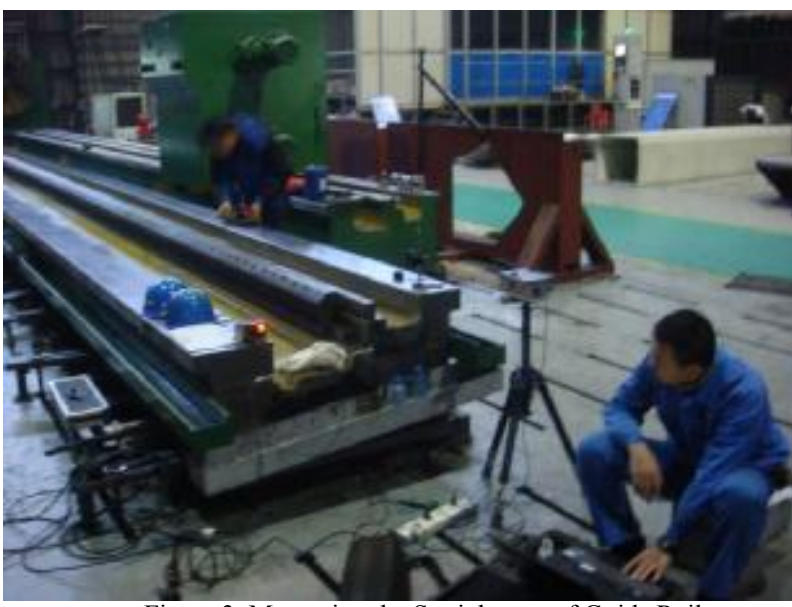

Figure.3 Measuring the Straightness of Guide Rail

The length of bridgeboard used in straightness measurement are $160 \mathrm{~mm}, 500 \mathrm{~mm}, 750 \mathrm{~mm}$ and $1000 \mathrm{~mm}$. Figure. 3 describes straightness measurement by Renishaw XL-80 laser interferometer. TABLE 1 describes the impact of different measurement tolerance $\delta$ to the measuring intervals that are derived.

TABLE 1 The Impact of Different Measurement Tolerance $\delta$ to the Measuring Intervals

\begin{tabular}{cc}
\hline Measurement tolerance & Theoretically optimal measuring interval $[\mathrm{mm}]$ \\
\hline$\delta=\mathrm{T} / 10$ & 544 \\
$\delta=\mathrm{T} / 8$ & 544 \\
$\delta=\mathrm{T} / 6$ & 620 \\
$\delta=\mathrm{T} / 5$ & 620
\end{tabular}

As can be seen from TABLE 1, the theoretically optimal measuring interval derived under the case of $\delta=T / 10$ is $544 \mathrm{~mm}$, so the raw data obtained by the $160 \mathrm{~mm}$ can be used to calculate as true values. TABLE 2 below describes the results of straightness measurement under the case of different measuring intervals by laser interferometer.

TABLE 2 Straightness under Different Measuring Intervals

\begin{tabular}{cc}
\hline Measuring interval $[\mathrm{mm}]$ & Straightness $[\mu \mathrm{m}]$ \\
\hline 160 & 40.922 \\
500 & 43.782 \\
750 & 33.8 \\
1000 & 26.846 \\
\hline
\end{tabular}

The theoretically optimal measuring interval derived is about $550 \mathrm{~mm}$, and the interval less than $550 \mathrm{~mm}$ can be used in practical. Taking the purpose of measuring efficiency and true expression of the straightness of the measured object into account, we can choose $500 \mathrm{~mm}$ as measuring interval. As can be seen from TABLE 2, the results of straightness measurement got under the case of interval $160 \mathrm{~mm}$ and $500 \mathrm{~mm}$ are basically the same, while the results are significantly smaller under the case of interval $750 \mathrm{~mm}$ and $1000 \mathrm{~mm}$, so the interval $500 \mathrm{~mm}$ can be the optimal measuring interval.

\section{IV.CONCLUSION}

This article proposes a calculation method for the largesize straightness measuring interval based on Nyquist sampling theorem. The results of validation experiments indicate that the method is feasible. According to the rail experimented, the interval $500 \mathrm{~mm}$ through theoretical derivation can be its optimal measuring interval. Because the measurement tolerance $\delta$ is from experiment in this paper, the subsequent research should give theoretical derivation and inspect the relationship between the measurement tolerance $\delta$ and measurement length.

\section{ACKNOWLEDGMENT}

This work is supported by the National Science and Technology Major Project of China (2013ZX04013-011-09) and Heilongjiang Postdoctoral Fund in 2013 (LBH-Z13209). The authors would like to express their appreciation to the contribution of Xiaozhong Lou and Zhonghe $\mathrm{Xu}$, who are the engineers at Qiqihar Heavy CNC Equipment CO., LTD, for offering help in experiments.

\section{REFERENCES}

[1] F.G. Huang. The Number of Measurement Extraction Points for Straightness Error Evaluation[J]. Journal of Huaqiao university: Natural Science Edition, 2011, 32(6): 615-617.

[2] F.G. Huang and Y.J. Zheng. Research straightness error measurement sampling scheme[J].Tool and Technology, 2007,10:95-98.

[3] X.F. Hou. Research of Sampling Spacing in Measuring Straightness Based on CMM[J].Tool and Technology, 2008, 42(6): 102-103.

[4] M. M. Dowling, P. M. Griffin, K.-L. Tsui and C. Zhou. Statistical Issues in Geometric Feature Inspection Using Coordinate Measuring Machines. Tchnometrics, 1997,39(1)3 24.

[5] A. Weckenmann, H. Eitzert, M. Garmer and H. Weber. FunctionalityOriented Evaluation and Sampling Strategy in Coordinate Metrology. Precision Engineering, 1995,17:244 252.

[6] Y.H Zheng, L.N. Zhang and K.W. Qing. Extraction scheme of the new generation GPS and its application research $[J]$. Machinery Design \& Manufacture, 2008 (6): 193-194.

[7] L.L. Huang, L.X. Wang, Yu Wang, H.L. Wang and F.G. Huang. Extraction of Straightness Error Signal with Harmonic Analysis. [J]. Journal of Huaqiao university: Natural Science Edition, ,2014,01:7-10.

[8] Z Li and Z.G. $\mathrm{Xu}$ and X.Q.Jiang. Interchangeability and Measurement Technology--Geometrical Product Specifications and Verification(Higher Education Press,China 2004). pp.67.

[9] B.Fei, Y.J. Fan and WenXiong Xu. Adaptive Sampling Method-A new method used in measuring the shape error[J]. Journal of Applied Sciences, 1994, 12(4): 423-430.

[10] F.X Zhang and H. Zhao. Theoretical research between sample step and deviation shape in straightness measurement $[\mathrm{J}]$. Aiavtion method \& measurement technology. 1995, 15(2): 9-11. 